\title{
Genetic deficiency of Wnt5a diminishes disease severity in a murine model of rheumatoid arthritis
}

Susan MacLauchlan ${ }^{1}$, Maria A. Zuriaga ${ }^{1}$, José J. Fuster ${ }^{1}$, Carla M. Cuda², Jennifer Jonason³, Fernanda Behzadi ${ }^{1}$, Jennifer Parker Duffen ${ }^{1}$, G. Kenneth Haines $1 I^{5}$, Tamar Aprahamian ${ }^{4}$, Harris Perlman ${ }^{2}$ and Kenneth Walsh ${ }^{1 *}$ (0)

\begin{abstract}
Background: Rheumatoid arthritis (RA) is a common autoimmune disease characterized by chronic inflammation of the joints, leading to bone erosion and joint dysfunction. Despite the recent successes of disease-modifying antirheumatic drugs (DMARDs), there is still clinical need for understanding the development and molecular etiology of RA. Wnts are developmental morphogens whose roles in adult pathology are poorly characterized. Wnt5a is a member of the non-canonical family of Wnts that modulates a wide range of cell processes, including differentiation, migration, and inflammation. Wnt5a has been implicated as a possible contributor to arthritis and it is upregulated in synovial fibroblasts from RA patients.
\end{abstract}

Methods: We investigated the role of endogenous Wnt5a in RA. Tamoxifen-inducible, Wnt5a knockout (Wnt5a cKO) mice and littermate controls were monitored for arthritis development and joint pathology using the K/BxN serum transfer-induced arthritis (STIA) model. To explore a role of Wnt5a in osteoclast fusion, bone marrow-derived monocytes (BMDMs) were differentiated in vitro.

Results: Wnt5a cKO mice were resistant to arthritis development compared to control littermates as assessed by ankle thickness and histologic measurements. Some parameters of inflammation were reduced in the Wnt5a cKO mice, including the extent of polymononuclear cell infiltration and extra-articular inflammation. Wnt5a cKO mice also exhibited less cartilage destruction and a reduction in osteoclast activity with concomitant reduction in tartrate-resistant acid phosphatase (TRAP), cathepsin K (CTSK), macrophage colony-stimulating factor (MCSF), matrix metalloproteinase (MMP)2 and MMP9 in the arthritic joints. Treatment of BMDMs with Wnt5a enhanced osteoclast fusion and increased the expression of dendrocyte-expressed seven transmembrane protein (DCSTAMP) and MMP9, that are necessary for osteoclast formation and activity.

Conclusions: These data suggest that Wnt5a modulates the development of arthritis by promoting inflammation and osteoclast fusion, and provide the first mouse genetic evidence of a role for endogenous Wnt5a in autoimmune disease.

Keywords: Rheumatoid arthritis, Wnt5a, Inflammation, Osteoclast fusion

\footnotetext{
* Correspondence: kxwalsh@bu.edu; kwalsh@theworld.com

${ }^{1}$ Molecular Cardiology, Whitaker Cardiovascular Institute, Boston University

School of Medicine, 700 Albany Street, W-611, Boston, MA 02118, USA

Full list of author information is available at the end of the article
} 


\section{Background}

Rheumatoid arthritis (RA) is a common chronic autoimmune disease that leads to significant morbidity and mortality. The prevalence of this disease is between 0.5 and $1 \%$ in the USA, with greater risk of incidence among women [1]. The molecular cues that trigger RA are complex and likely require a combination of genetic and environmental factors. Ultimately, the loss of self-tolerance to self-proteins, typically citrullinated-proteins, results in disease progression through inflammation, synovial hyperplasia, and osteoclast activation [2]. These processes lead to the recognizable hallmarks of the disease, including joint swelling, pain, and dysfunction. Although joint involvement is the major clinical manifestation of the autoimmune process, RA patients suffer from a number of co-morbidities including increased risk of cardiovascular disease even when accounting for the increased inflammatory state [3, 4]. Significant strides have been made in the treatment of RA. Standard therapy for RA includes methotrexate and one of several disease-modifying anti-rheumatic drugs (DMARDs) that target the key inflammatory molecules that promote RA, including TNF $\alpha$ (infliximab, etanercept) and IL6 (tocilizumab). These treatments allow many patients to achieve disease-free status, but their use is associated with significant side effects and these biologic agents do not confer sustained remission after drug cessation $[1,5]$. Thus, there remains a clinical need for improved understanding of novel targets for drugs in the process of RA development.

The Wnt family proteins are developmental regulators that are becoming appreciated for their diverse roles in adult physiology. Wnt5a is the archetypal non-canonical Wnt, the subset of Wnts whose functions are independent of beta-catenin [6]. In post-natal tissue, the functions of Wnt5a are diverse and include modulation of cell fate decisions and inflammation. Previous work has established Wnt5a as a pro-inflammatory molecule in a number of settings including vascular dysfunction [7, 8], peripheral arterial disease [9], and cardiometabolic disease [10-12]. Wnt5a has also been demonstrated to promote expression of various cytokines that may contribute to RA progression [13-16]. However, studies on the role of Wnt5a in immune regulation can be confounded by the likely presence of TLR4 agonists in commercial Wnt5a protein preparations [17]. Thus, analyses of Wnt5a function using mouse genetic models are warranted as they are not subject to this potential confounder.

Wnt5a is increased in the synovium in patients with RA and osteoarthritis (OA) [18], and inhibition of Wnt5a is reported to block the activation of cultured synovial fibroblasts from RA patients [19]. In addition, the delivery of a soluble form of receptor-tyrosine orphan receptor 2 (ROR2), a candidate decoy receptor for
Wnt5a, leads to reduced radiographic severity in mice undergoing collagen-induced arthritis (CIA) [20]. These data underscore the potential clinical value of Wnt5a in the pathogenesis of RA. However, there is as yet no direct genetic evidence to support that endogenous Wnt5a plays a contributory role in the development of RA.

In the present study, we used inducible Wnt5a knockout (Wnt5a cKO) and littermate control mice in the $\mathrm{K} / \mathrm{BxN}$ serum transfer-induced arthritis (STIA) model. Wnt5a cKO mice were protected from development of RA, both in terms of ankle thickness and other markers of disease severity. Histologic examination demonstrated reduced inflammation in the Wnt5a cKO, including reduced PMN infiltrate into the inflamed joints. Less osteoclast activity was also observed in the arthritic Wnt5a cKO joints compared to the controls. Mechanistically, this may result from decreases in osteoclast fusion as Wnt5a promoted osteoclast fusion in an in vitro culture of bone marrow-derived macrophage (BMDM) differentiation. Wnt5a increased the expression of critical molecules for osteoclast differentiation and fusion, specifically dendrocyte-expressed seven transmembrane protein (DCSTAMP) and matrix metalloproteinase 9 (MMP9). These data suggest Wnt5a signaling as a modulator of inflammation and osteoclast fusion could represent a component of disease activity in RA patients.

\section{Methods}

\section{Animals}

All animal protocols were approved by the Institutional Animal Care and Use Committee (IACUC), Boston University School of Medicine (BUSM). Wnt5a whole body inducible cKO mice (Wnt5a cKO) were generated as previously described [10] by selective breeding of the Wnt5a floxed mice [21] with the $U B C$ cre promoter mice (Jackson Labs, Bar Harbor, ME, USA, stock number 007001). Female littermate $W n t 5 a$ floxed cre-negative mice (control) and cre-positive mice (Wnt5a cKO) were used for this study. Wnt5a recombination was induced in the mice at 6 weeks of age by five daily intraperitoneal injections of tamoxifen delivered in corn oil (80ug tamoxifen per $\mathrm{g}$ body weight each day). Both control and Wnt5a cKO mice were treated with tamoxifen. Baseline mice (at least four per genotype) were euthanized following 2 weeks of tamoxifen wash out. Tissue was collected and processed as described in the following section. Mice were used in the arthritis model following 2 weeks of tamoxifen wash out ( $n=13$ per genotype for RA studies). Female C57BL6) (Jackson Laboratories) mice 10-12 weeks of age were used for the in vitro fusion assays $(n=3)$.

\section{$\mathrm{K} / \mathrm{BxN}$ serum transfer-induced arthritis (STIA) model}

Rheumatoid arthritis development was induced in control and Wnt5a cKO mice using the $\mathrm{K} / \mathrm{BxN}$ serum 
transfer model of arthritis as previously described [22]. Briefly, serum from $\mathrm{K} / \mathrm{BxN}$ mice (the offspring of mice expressing the KRN $\mathrm{T}$ cell receptor transgene and the major histocompatibility complex (MHC) class II molecule $\mathrm{A}(\mathrm{g} 7)$, which develop spontaneous arthritis) was isolated at 8 weeks of age. Passive transfer of arthritis was induced in control and Wnt5a cKO mice by intraperitoneal injection of $150 \mu \mathrm{l}$ of $\mathrm{K} / \mathrm{BxN}$ serum at day 0 (d0) and d2. Severity of arthritis was monitored by two measures prior to serum injection and every other day for 28 days. The clinical score of each joint was determined using a well-established scoring metric [22-24]. The reported clinical score is the summation of the scores for all four appendages. Ankle thickness was measured at the thickest point of the ankle using digital Vernier calipers (Fine Science Tools). Significance for the clinical score and ankle thickness measurements was determined using the statistical package in Prism GraphPad using two-way analysis of variance (ANOVA). Significance was determined using the Sidak post-hoc test. Tissues were collected at $\mathrm{d} 7$ ( $n=6$ per genotype) or $\mathrm{d} 28(\mathrm{n}=7$ per genotype) following induction of arthritis. Serum was collected by cardiac puncture after euthanasia by carbon dioxide inhalation. Paw joints were snap frozen for mRNA isolation and ankle joints were fixed in 10\% formalin and decalcified in 0.375 M EDTA.

Paw joints were pooled for each individual animal prior to mRNA extraction. The joints were cleaned of skin, homogenized with Qialyzer (Qiagen) and mRNA was isolated with the Qiagen RNeasy Fibrous tissue kit according to the manufacturer's directions. cDNA was transcribed from $1 \mu \mathrm{g}$ of RNA using High Capacity cDNA Kit (Applied Biosystems) and quantitative PCR (qPCR) was performed using SYBR-based detection (primer sequences are shown in Table 1). Fold changes were calculated after normalization of the average hypoxanthine guanine phosphoribosyl transferase (HPRT) and beta-actin relative to baseline mice. $P$ values $\leq 0.05$ from

Table 1 Sequences for SYBR qPCR

\begin{tabular}{|c|c|c|}
\hline Gene ID & Forward primer & Reverse primer \\
\hline$\overline{C A R 2}$ & TCC CAC CAC TGG GGA TAC AG & CTC TTG GAC GCA GCT TTA TCA TA \\
\hline CD68 & TGT CTG ATC TTG CTA GGA CCG & GAG AGT AAC GGC CTT TाT GTG A \\
\hline CLCN7 & CGC CAG TGT CAT TCT GCA CT & GCT TCT CGT TGT GTG GAA TCT \\
\hline CTSK & GAA GAA GAC TGA CCA GAA GCA G & TCC AGG TTA TGG GCA GAG ATT \\
\hline DCSTAMP & GGG GAC TTA TGT GTT TCC ACG & ACA AGG CAA CAG ACT CCC AAA T \\
\hline$F 4 / 80$ & CTT TGG CTA TGG GCT TCC AGT C & GCA AGG AGG ACA GAG TाT ATC GTG \\
\hline GAPDH & TGA CCA CCA TGG AGA AGG C & GCT AAG CAG TTG GTG GTG CA \\
\hline HPRT & TCA GTC AAC GGG GGA CAT AAA & GGG GCT GTA CTG CTT AAC CAG \\
\hline$I L 1 \beta$ & TGA CAG TGA TGA GAA TGA CCT GTT C & TTG GAA GCA GCC CTT CAT CT \\
\hline $1 / 6$ & GCT ACC AAA CTG GAT ATA ATC AGG A & CCA GGT AGC TAT GGT ACT CCA GAA \\
\hline MCP1 & GCC TAC TCA TTG GGA TCA TCT TG & CAG CCA GAT GCA GTT AAC GC \\
\hline MCSF & GGC TTG GCT TGG GAT GAT TCT & GAG GGT CTG GCA GGT ACT C \\
\hline MMP2 & CAG GGT GGT GGT CAT AGC TAC TT & GAG ACT TTG GTT CTC CAG CTT CA \\
\hline MMP9 & CAA GTG GGA CCA TCA TAA CAT CA & CTC GCG GCA AGT CTT CAG A \\
\hline MMP14 & AGT GAC AGG CAA GGC TGA TाT & AGG GGT GTA ATT CTG AAT GCA G \\
\hline NFATCl & CAG GGC GAG TTC GAC TTC G & TGA CAC TAG GGG ACA CAT AAC TG \\
\hline$O P G$ & ACC CAG AAA CTG GTC ATC AGC & CTG CAA TAC ACA CAC TCA TCA CT \\
\hline OSCAR & CCT AGC CTC ATA CCC CCA G & CGT TGA TCC CAG GAG TCA CAA \\
\hline RANKL & CAG CAT CGC TCT GTT CCT GTA & CTG CGT TIT CAT GGA GTC TCA \\
\hline RANK & GGA CGG TGT TGC AGC AGA T & GCA GTC TGA GTT CCA GTG GTA \\
\hline RCAN2 & CCA CTC TGG TCG CCT GTG T & CGG AAC AGT CCC TCG AAT TाT TCC TTA \\
\hline TBP & CTT CCT GCC ACA ATG TCA CAG & СCT TTC TCA TGC TTG CTT CTC TG \\
\hline$T N F a$ & CGG AGT CCG GGC AGG & GCT GGG TAG AGA ATG GAT GAA \\
\hline TRAP & CAC TCC CAC CCT GAG ATT TGT & CAT CGT CTG CAC GGT TCT G \\
\hline Wnt5a Ex2 & CAA ATA GGC AGC CGA GAG AC & CTC TAG CGT CCA CGA ACT CC \\
\hline 185 & AAT CAA GAA CGA AAG TCG GAG G & GCG GGT CAT GGG AAT AAC G \\
\hline $36 B 4$ & GCT CCA AGC AGA TGC AGC A & CCG GAT GTG AGG CAG CAG \\
\hline
\end{tabular}


the $t$ test calculated using GraphPad Prism were considered significant.

\section{Histopathologic assessment}

Paraffin-embedded tissue sections were stained with hematoxylin and eosin (H\&E). Disease severity was analyzed by a pathologist (KGH) blinded to the genotypes as previously described [25] ( $\mathrm{n}=6$ per genotype). Clinical score, cartilage destruction, bone erosion, inflammation, polymononuclear (PMN) infiltration, extra-articular inflammation, lymphocyte infiltration, pannus formation and synovial lining average were evaluated as previously described [23, 25-28]. Tartrate acid resistant phosphatase (TRAP) staining was performed as specified by the manufacturer (Sigma Kit) ( $n=6$ per genotype at d7 and at least 4 per genotype for baseline analysis). Stained sections were visualized on a Keyence BZ-X700 and analyzed with the on-board image processing software to identify total TRAP + area in at least four fields per mouse in both ankle joints.

\section{L cell conditioned media}

L control cells (ATCC, CRL-2648) and L Wnt5a cells (ATCC, CRL-2814) were used to generate controls and Wnt5a conditioned media as previously described [29]. L cells were maintained in DMEM with 10\% FBS and pen/strep/Lglut. Conditioned medium for the fusion assays were generated in complete alpha-MEM with $10 \%$ FBS and pen/strep/Lglut. This conditioned medium was diluted 1:4 with fresh complete alpha-MEM medium for the fusion assays.

\section{In vitro analysis of fusion}

BMDM were isolated from female C57BL6J mice (Jackson Laboratories) and cultured in alpha-MEM with $10 \%$ FBS and pen/strep/Lglut. Following differential plating to purify monocyte cells in the presence of macrophage colony-stimulating factor (MCSF) $(100 \mathrm{ng} / \mathrm{mL}), 10^{\wedge} 5$ cells per well were plated in 24 well plates in the presence of diluted L cell control or diluted Lcell Wnt5atreated medium. BMDM were induced to fuse with the stimulation cocktail (MCSF $(50 \mathrm{ng} / \mathrm{mL}$ ) and RANKL $(50 \mathrm{ng} / \mathrm{mL})$ ), which was replenished at $\mathrm{d} 2$ and $\mathrm{d} 4$. RNA was isolated at $\mathrm{d} 2$ and $\mathrm{d} 5$ using the miRNeasy Micro Kit (Qiagen) and $1 \mu \mathrm{g}$ of RNA was transcribed using the Quantitect kit (Qiagen). SYBR chemistry was used to detect transcripts and these were analyzed on a ViiA7 using the primers listed in Table 1. Significance was determined in GraphPad Prism using two-way ANOVA with the Tukey post-hoc test, considering $p$ values $\leq 0.05$ as significant. To enumerate osteoclasts, d5 cultures were stained for TRAP (Sigma) and co-stained with 4',6-diamidino-2-phenylindole (DAPI) (Thermo Fisher Scientific). Cultures were visualized using a Keyence microscope. Osteoclasts were identified as TRAP+ multinucleated cells (3 nuclei or more). Total nuclei, total TRAP+ cells, total osteoclasts and total nuclei in osteoclasts were enumerated in at least six random high powered fields per condition per experiment. The osteoclast frequency per 1000 cells was calculated as the ratio of total osteoclasts per high-powered field (HPF) divided by total nuclei in the same HPF. The percentage of cells in osteoclasts is the total nuclei in osteoclasts per HPF divided by the total nuclei in the same image. Statistical significance for enumeration tests was performed using Prism Software and the unpaired $t$ test and considering significance at $p \leq 0.05$. All in vitro studies were performed three times. Enumeration studies were performed in duplicate three times.

\section{Results}

Wnt5a-deficiency attenuates the development of RA-like disease

Due to the association of Wnt5a with clinical arthritis, we sought to determine its role in the murine STIA model of RA. In apparent contrast to clinical arthritis [18], Wnt5a transcript levels were reduced at d7 after RA induction (Additional file 1: Figure S1). However, active RA induced the levels of the Wnt5a co-receptor ROR2 mRNA at this time point (Additional file 1: Figure S1). To further evaluate the role of Wnt5a in the development of RA, a murine model of Wnt5a deficiency was subjected to the STIA model. Using primers that hybridize within the floxed Exon 2 of Wnt5a (Additional file 1: Figure S2A), the Wnt5a cKO mice exhibited $>90 \%$ decrease in Wnt5a in the paws of Wnt5a cKO mice after 2 weeks of tamoxifen-induced ablation (Additional file 1: Figure S2A) compared with littermate controls. Baseline measures in healthy Wnt5a cKO mice did not demonstrate alterations in inflammatory markers (Additional file 1: Figure S2B), in ankle morphology (Additional file 1: Figure S3), or in osteoclast activation (Additional file 1: Figure S4). Following STIA induction of RA, there was attenuated development of arthritis in the Wnt5a cKO mice in terms of ankle thickness, which plateaued below the thickness developed in the littermate controls and resolved in a similar timeframe (Fig. 1a). In a separate cohort of animals, the arthritic process was examined in control and Wnt5a cKO mice at the earliest peak of inflammation (d7). Measurement of the disease severity was performed by a pathologist blinded to genotype using previously established histopathologic scoring metrics $[23,24]$, using $H \& E$ stained sections from control and Wnt5a cKO mice at d7 (Fig. 2a). The Wnt5a cKO mice displayed statistically significant reduction in overall disease severity (overall H\&E score) and this cohort exhibited a significant reduction in clinical score (Fig. 2b). Histologic analysis of the arthritic joints also revealed that Wnt5a cKO mice exhibit significant 


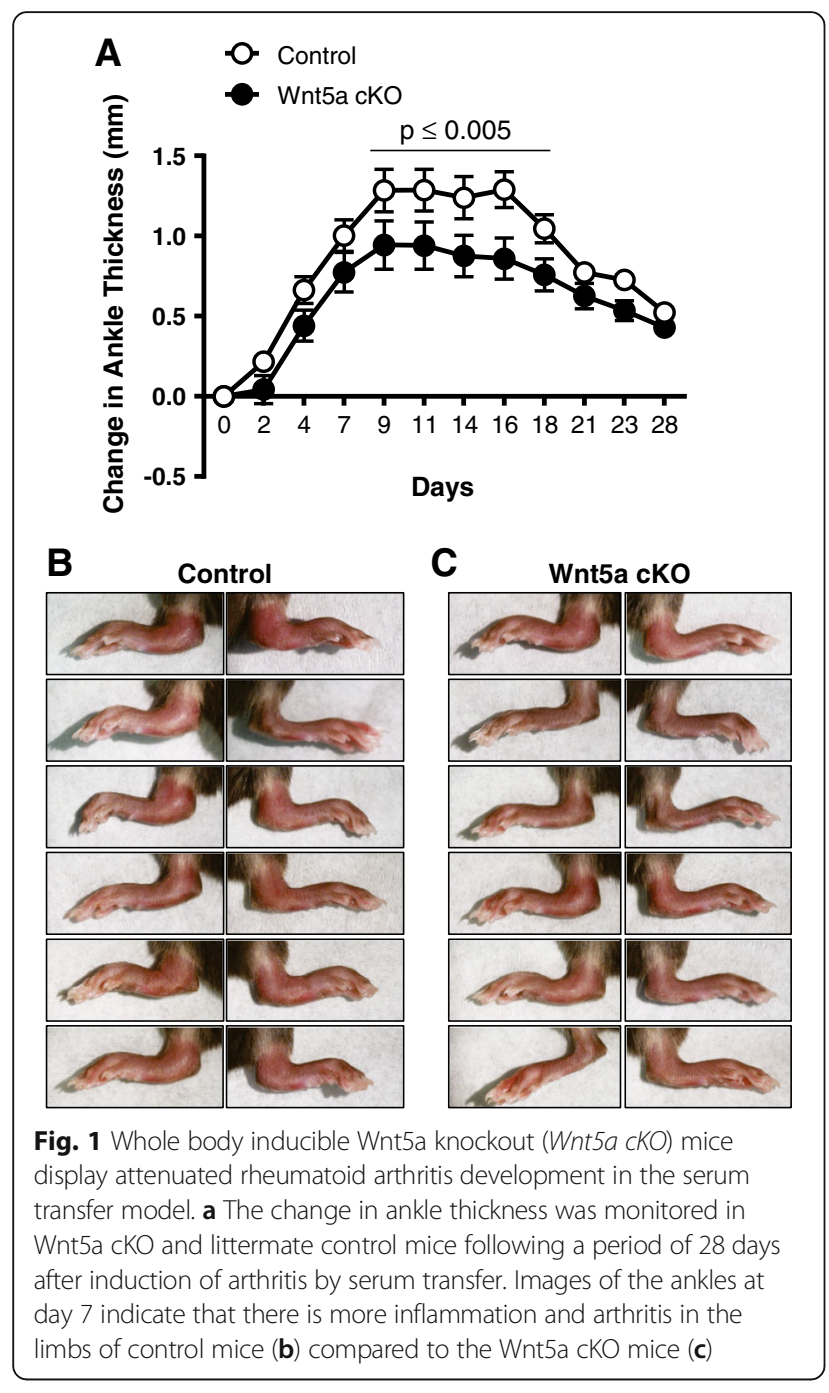

reductions in some parameters of inflammation, including measures of reduced extra-articular inflammation and PMN infiltration (Fig. 2b). Although not statistically different, trends of reduced pannus formation, synovial lining average and bone erosion were also observed in the Wnt5a cKO mice compared to control mice (Fig. 2b and Additional file 1: Figure S5). Numerous studies have reported that Wnt5a promotes cytokine expression [1316]. However, only trends of decreased TNF $\alpha, I L 6$, and IL1 $\beta$ were observed in Wnt5a cKO at day 7 following RA induction (Additional file 1: Figure S6A), which were not statistically significant.

\section{Decreased osteoclast activity in arthritic Wnt5a cKO mice}

We next evaluated the osteoclast activity in the arthritic ankles from control and Wnt5a cKO mice. TRAP staining (Fig. 3a) demonstrated robust activity in the control mice, with obvious staining at multiple destruction pits, whereas Wnt5a cKO mice displayed a markedly reduced TRAP-positive area as quantified in Fig. 3b. Consistent with these findings, decreased levels of TRAP and cathepsin $K$ (CTSK) mRNA were detected in the arthritic paws of Wnt5a cKO mice (Fig. 3c), but there were no changes in the calcitonin receptor $(C A L)$ or carbonic anhydrase 2 (CAR2) mRNA in Wnt5a cKO mice (Additional file 1: Figure S6B). There was a trend toward lower expression of the osteoclast differentiation signal RANKL and reduction in the expression of MCSF observed in the arthritic paws of Wnt5a cKO mice (Fig. 3c). Consistent with their roles both in inflammation and in osteoclast activity, levels of MMP2 and MMP9 mRNA were significantly decreased in the arthritic paws of Wnt5a cKO mice (Fig. 3c).

\section{Wnt5a promotes osteoclast fusion in vitro}

To attain a deeper understanding of the mechanism of Wnt5a action in the process of osteoclastogenesis, in vitro analyses were performed to determine whether Wnt5a has an intrinsic ability to promote osteoclast fusion. MCSF-dependent BMDM were stimulated to fuse in the presence of $\mathrm{L}$ cell medium conditioned by cells that were engineered to overexpress Wnt5a (Wnt5a) or control media (conditioned by L cells without Wnt5a overexpression). TRAP-positive (dark color) multinucleated osteoclasts formed after 5 days of RANKL stimulation (Fig. 4a). Osteoclast fusion was stimulated by Wnt5a, both in terms of total incidence (osteoclast frequency per 1000 cells) and in the percent of nuclei fused into osteoclasts (Fig. 4b). In contrast, Wnt5a did not significantly change the nuclei per osteoclast total or total nuclei in osteoclasts (Additional file 1: Figure S7).

Transcripts were analyzed in fusing BMDM cultures after 2 and 5 days of RANKL stimulation to further evaluate the impact of Wnt5a on osteoclast fusion. Wnt5a mRNA levels were not altered during the fusion process (Additional file 1: Figure S8). Treatment with Wnt5a did not significantly alter the expression of osteoclast genes (CTSK or TRAP) or an inhibitor of osteoclastogenesis (OPG) (Additional file 1: Figure S8). However, Wnt5a promoted the expression of DCSTAMP (Fig. 4c), a cell surface molecule that is essential for osteoclast fusion [30]. Consistent with observations in the STIA model, fusing macrophages treated with Wnt5a expressed increased levels of $M M P 9$ but not $M M P 2$ (Fig. 4d). In contrast, Wnt5a did not affect the expression of other nuclear factor activating $\mathrm{T}$ cells $1 \mathrm{c}$ (NFATc1) downstream genes, including MMP14, CLCN7, CAR2, RCAN2 and OSCAR, or NFATC1 itself (Additional file 1: Figure S9).

\section{Discussion}

Wnt5a is elevated in the synovium of RA patients [18], and enhanced Wnt5a secretion by fibroblast-like synoviocytes has been attributed to their persistent activation 

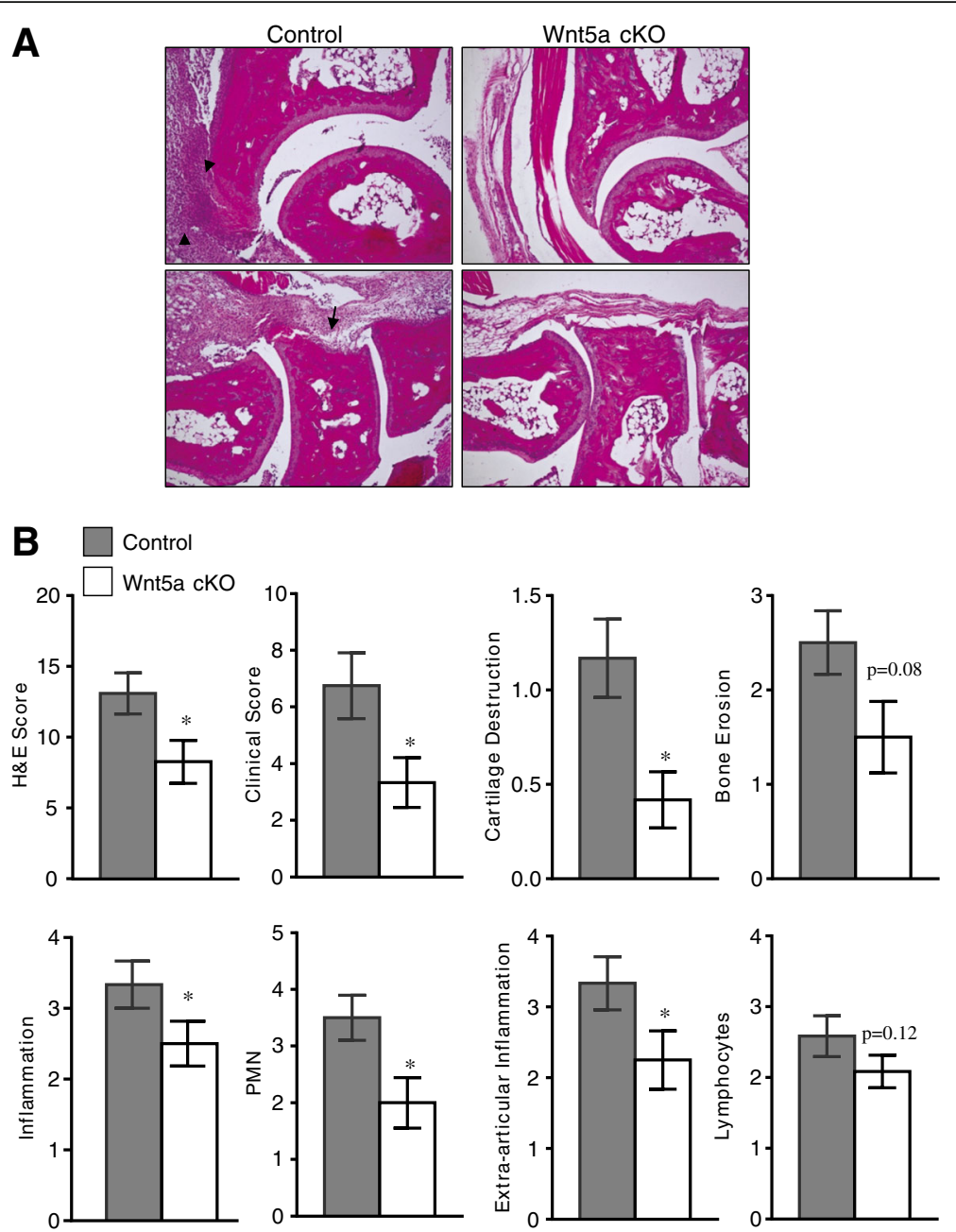

Fig. 2 Whole body inducible Wnt5a knockout (Wnt5a cKO) mice develop less severe rheumatoid arthritis (RA) based upon histological analyses. a H\&E-stained sections from the ankles in control and Wnt5a cKO mice at day 7 after induction of arthritis demonstrate hallmark features of the disease at the tarsotibial joint (top panels) and the tarsonavicular joint (bottom panels), including expanded pannus formation (arrowheads) and destruction pits (arrow). b Qualitative metrics of the damage induced by RA in control and Wnt5a cKO mice demonstrates reduced overall disease severity (H\&E score), clinical score, cartilage destruction, inflammation, polymononuclear (PMN) cell infiltration and extra-articular lesions in the joints of Wnt5a cKO mice. ${ }^{*} P \leq 0.05$

[19]. Thus, we sought to provide genetic evidence for a causal link between Wnt5a and RA development. The present study utilized a mouse model in which ablation of Wnt5a is inducible, thereby avoiding the embryonic lethality of the conventional knockout strain [21]. This inducible ablation model also permits the ablation of Wnt5a proximal to the induction of arthritis, thereby eliminating the confounding effects of Wnt5a in bone development [20] (Additional file 1: Figure S1-S3). Collectively, this study shows that mice deficient for Wnt5a are resistant to development of RA-like disease in the STIA model.

The acute genetic ablation of Wnt5a in the STIA model led to reductions in some markers of inflammation. Based upon histologic analysis, Wnt5a cKO mice exhibited statistically significant reductions in overall inflammatory status, extra-articular inflammation and PMN cell infiltration (Fig. 2). In this regard, it is wellappreciated that neutrophils are important mediators in the development of arthritis [31, 32]. Failure to recruit neutrophils correlates with reduction in the overall progression of disease severity in other models of arthritis [33]. Further, since Wnt5a is described to be chemotactic to neutrophils [34], it is possible that this is a significant component of the mechanism by which Wnt5a cKO mice are protected from the development of STIA.

Using the Wnt5a cKO mouse model, we recently reported that Wnt5a ablation protects against adipose tissue inflammation and systemic metabolic dysfunction that is associated with diminished levels of monocyte chemoattractant protein 1 (MCP-1), IL6 and TNF $\alpha$ in the adipose tissue [10]. Although these cytokines are 
A
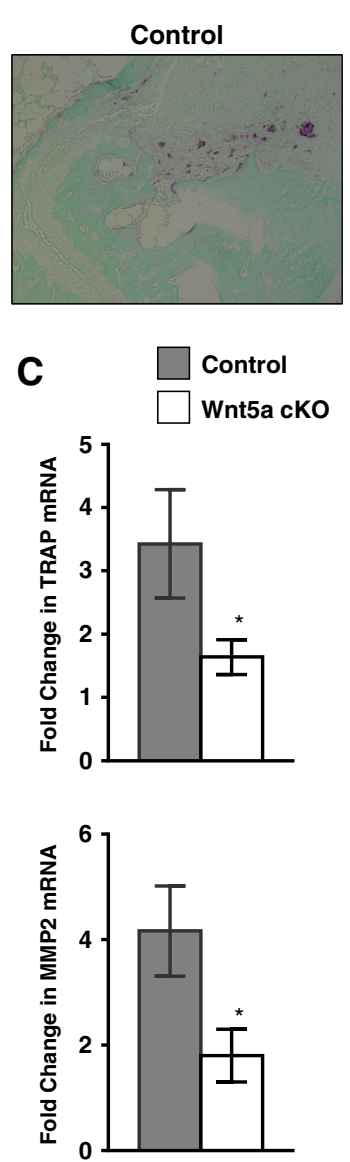
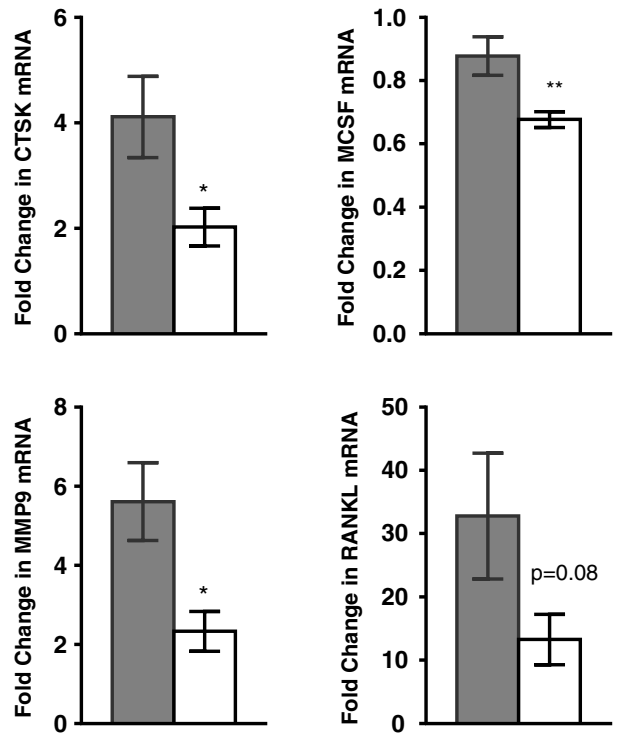

B
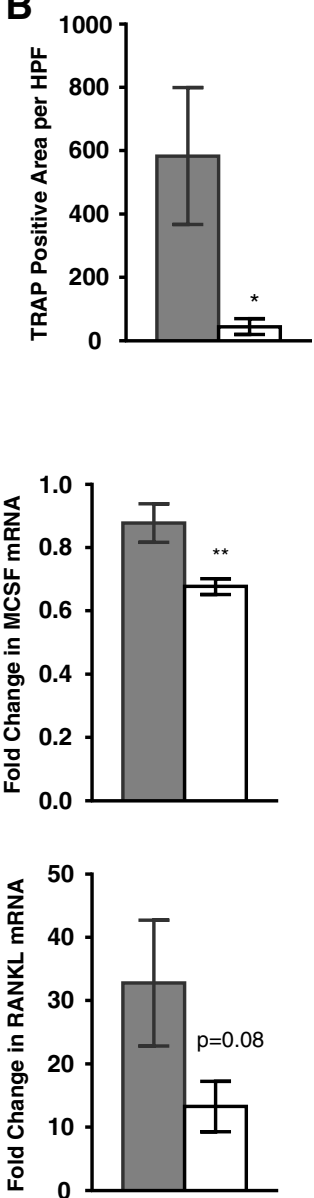

Fig. 3 Whole body inducible Wnt5a knockout (Wnt5a cKO) mice have reduced osteoclast activity after induction of arthritis. a Tartrate-resistant acid phosphatase (TRAP) staining, indicated by purple color in control (left panel) and Wnt5a cKO (right panel), indicates fewer areas of osteoclast activity in the Wnt5a cKO mice at day 7. b Quantification of the percent of TRAP-positive area revealed reduced osteoclast activity in the Wnt5a CKO (white bars) compared to the controls (gray bars). c The levels of mRNA for the TRAP, Cathepsin K (CTSK), macrophage colony-stimulating factor (MCSF), matrix metalloproteinase 2 (MMP2) and MMP9 were reduced in the paws from the arthritic Wnt5a cKO mice compared to the arthritic controls. ${ }^{*} P \leq 0.05$. A trend toward reduced RANKL transcript was noted

shared in the pathogenesis of metabolic dysfunction and RA $[1,5]$, only trends towards reduction of $T N F \alpha, I L 6$, and $I L 1 \beta$ transcripts were observed in the arthritic paws from the Wnt5a cKO mice (Additional file 1: Figure S6) as was a trend toward diminished lymphocyte infiltration in the histological sections (Additional file 1: Figure S5). In total, these data are indicative that Wnt5a is one of many modulators of inflammation in the RA model, which can be compensated for by other inflammatory molecules. However, there appears to be a modest and appreciable protection from the inflammation in RA conferred by Wnt5a deficiency. Further, we acknowledge the possibility that Wnt5a has a more profound impact on inflammation in the STIA model at other time points.

In contrast to the increase in Wnt5a expression in the synovium of RA patients [18], we found a reduction in the amount of Wnt5a in the joints from mice undergoing STIA. Beyond species differences, there are a number of possible explanations for this discrepancy. First, there was a concurrent increase in the Wnt5a coreceptor ROR2 (Additional file 1: Figure S1), indicating that reduced amounts of Wnt5a might be sufficient to drive RA pathology. In this regard, Wnt5a is highly glycosylated and is believed to exhibit its effects through short-range paracrine interactions [35, 36]. Thus, localized changes in Wnt5a may be sufficient to modulate RA development, particularly when paired with a concomitant increase in ROR2. It should be also noted that our analysis was performed using the entire joint; including fibroblast-like synoviocytes, bone, bone marrow, and extra-articular inflammatory tissue. Indeed, although Wnt5a is reported to be among the most highly expressed Wnt homologs expressed in bone, immunohistochemical analysis demonstrated that it is predominantly expressed 
A

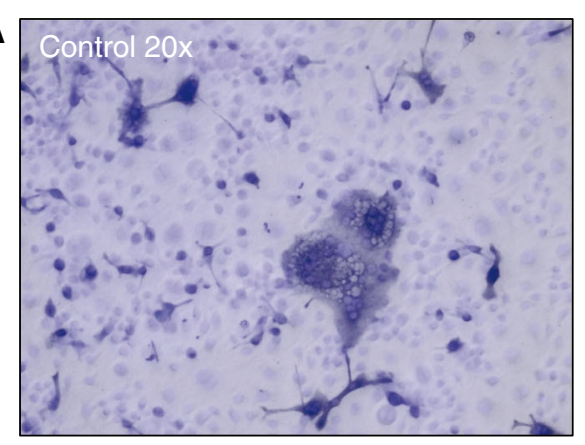

B

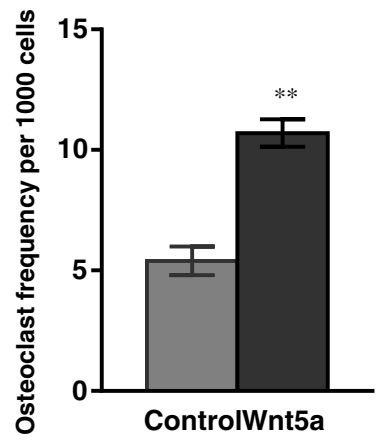

C

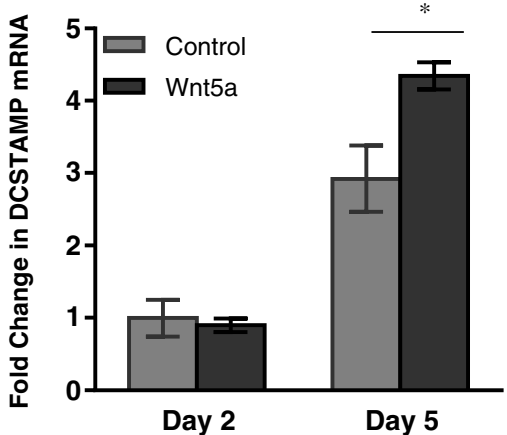

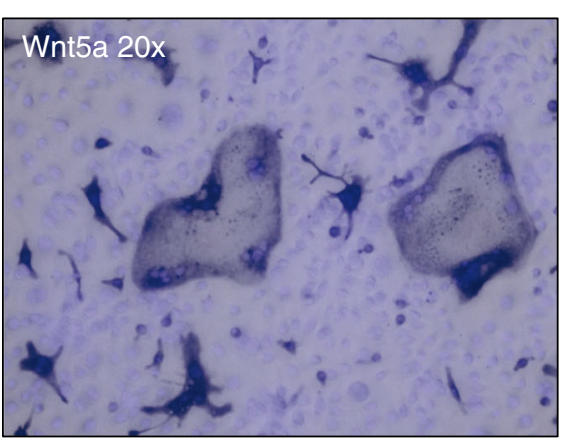
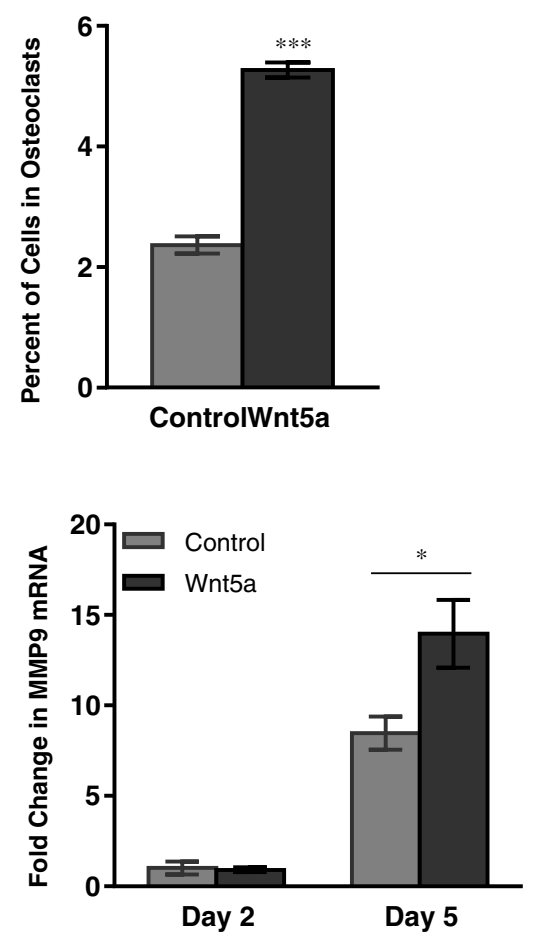

Fig. 4 Wnt5a promotes osteoclast formation through enhanced dendrocyte-expressed seven transmembrane protein (DCSTAMP) and matrix metalloproteinase 9 (MMP9) expression. Osteoclasts were formed in vitro from bone marrow-derived monocytes in $L$ cell conditioned medium (Control) or L Wnt5a conditioned medium (Wnt5a). Osteoclasts were visualized after 5 days of RANKL stimulation by tartrate-resistant acid phosphatase (TRAP) (dark color) and 4',6-diamidino-2-phenylindole (a). Osteoclasts were enumerated by identifying TRAP+ cells with three or more nuclei and expressed as frequency per 1000 cells or by percent of nuclei in osteoclasts (b). By both measures, Wnt5a increased the osteoclast formation ( $\left.{ }^{* *} p \leq 0.01,{ }^{* * *} p \leq 0.001\right)$. c mRNA isolated from fusing osteoclast cultures at day 2 and day 5 were analyzed for DCSTAMP and MMP9 expression. The levels were increased by Wnt5a treatment. ${ }^{*} P \leq 0.05$ for comparison of control and Wnt5a-treated cultures

by osteoblast/osteoclasts and not the osteocyte [20]. Thus, further exploration of Wnt5a in the arthritic space, beyond the synovial tissue, is warranted.

Wnt5a has been functionally implicated in the development of RA by a study that delivered a soluble version of ROR2, a putative Wnt5a receptor, to mice in the collagen-induced arthritis (CIA) model [20]. Although mice receiving the soluble ROR2 were not protected from development of ankle thickness or clinical score, they did exhibit reduced radiographic damage [20], consistent with a reduction in osteoclast activity. Our work supports and extends these observations in several ways.
First, using the Wnt5a cKO mouse model we provide direct mouse genetic evidence to support that Wnt5a plays a role in the pathogenesis of RA. Second, the inducible Wnt5a cKO mice were used to ablate Wnt5a immediately prior to the induction of the STIA model, which eliminates the confounding effects of Wnt5adeficiency on long-term bone development [20, 37-39]. Third, we document an effect of Wnt5a in the STIA model, which recapitulates the initiating events of the disease with a higher penetrance than the CIA model. Notably, the STIA model also avoids the use of adjuvant that can potentially act as a confounder. Finally, our 
study provides mechanistic detail about the role of Wnt5a in osteoclast fusion.

Macrophage cell fusion is required for the formation of multinucleated osteoclasts that function in the catabolism of bone matrix. Osteoclast formation from myeloid precursors is regulated by a sequence of molecular events involving the cell surface receptors including DCSTAMP, CD36, CD47, TREM2 and E-cadherin [40]. Additionally, osteoclast-specific genes including MMP9, CTSK and TRAP [41], are also required for cell fusion during osteoclastogenesis. Although it has been implicated in differentiation of multiple cell types, Wnt5a is not widely appreciated to promote cellular fusion. In the present study, we find that exogenous Wnt5a added to fusing BMDM enhanced their fusion into osteoclasts and upregulated DCSTAMP (Fig. 4), a cell surface molecule essential for formation of osteoclasts in vivo [30]. $M M P 9$ was also found to be upregulated by Wnt5a in fusing osteoclasts (Fig. 4c). MMP9 has been shown to be critical for the development of arthritis as MMP9 KO mice develop less inflammation and joint destruction in the serum transfer model [42]. MMP9 has also been shown to modulate several aspects of osteoclast function, including enhancement of cell migration, potentiation of cell fusion, and increased bone metabolism [43-46]. Altogether, our data indicate that Wnt5a promotes osteoclast formation, and suggests a possible role of Wnt5a in development of other bone resorption disorders.

Rheumatoid arthritis is associated with cardiometabolic diseases [3, 4], and a growing number of animal studies have investigated this linkage. Assessment of arthritis development in ApoE-deficient mice using the CIA model has led to conflicting reports on the consequences of hypercholesterolemia on promoting arthritis $[47,48]$. However, using a modified chronic STIA model, more recent evidence demonstrated that ApoE-deficient mice display aggravated development of arthritis but not an increased plaque burden [49]. Conversely, when placed on an atherogenic diet, $\mathrm{K} / \mathrm{BxA}^{\mathrm{g} 7}$ mice display increased plaque burden [50]. The present study suggests that Wnt5a might be a possible common mechanism linking both RA and cardiovascular disease. It has been shown that Wnt5a contributes to increased cardiac dysfunction following ischemia/reperfusion injury [11] and to impaired revascularization in a model of peripheral arterial disease [9]. Moreover, previous research demonstrated that Wnt5a impairs metabolic function at least in part through its role in exacerbating adipose tissue inflammation in the obese state $[10,12]$. Wnt5a is markedly upregulated in visceral adipose tissue (VAT) and correlates strongly with indices of inflammation and diabetes mellitus $[10,51]$. Despite the existence of "rheumatoid cachexia" in late-stage disease, large scale meta-analysis reveals that obesity is a risk factor for RA that is associated with disease severity [52, 53]. It has been proposed that adipokines, cytokines secreted by adipose tissue, are molecular drivers of both diseases [52, 54]. RA patients exhibit elevated adipokine levels, including leptin, adiponectin, and resistin, in serum or synovial fluid compared to healthy patients [52, 55-57], and these factors frequently correlate with disease progression [58, 59]. Collectively, these observations warrant further study to establish whether Wnt5a functions as a putative nexus target in RA and cardiometabolic diseases.

Recent advances in DMARDs have revolutionized the treatment landscape for RA patients. However, cessation of treatment inevitably leads to relapse. Furthermore, a subset of patients remains refractory to these therapeutics agents. Current treatment modalities focus on inhibition of the inflammatory process and do not directly target osteoclast activity. Based upon the multi-modal influence of Wnt5a on RA etiology, specifically as a modulator of osteoclast activity and inflammation, it may represent a novel target that has potential to modulate both arms of RA disease progression.

\section{Conclusions}

Using a genetic model, this study demonstrates for the first time that loss of Wnt5a attenuates the development of RA. Wnt5a functions in two major aspects of RA pathology; through enhancement of some parameters of inflammation and by promoting osteoclast fusion. These results are the first to demonstrate a role of Wnt5a in autoimmune disease progression or in cell-cell fusion.

\section{Additional file}

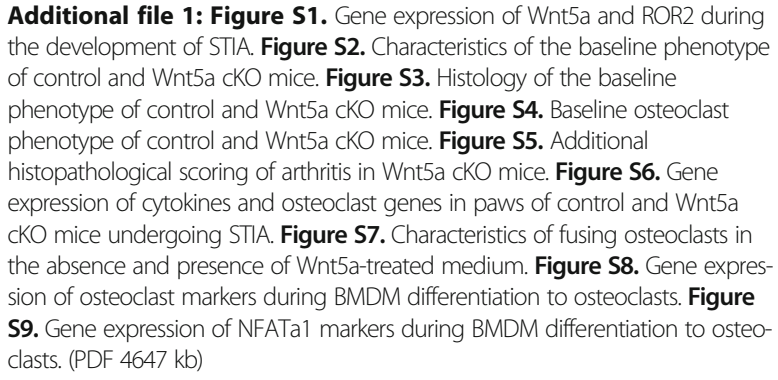
the development of STIA. Figure S2. Characteristics of the baseline phenotype of control and Wnt5a cKO mice. Figure S3. Histology of the baseline phenotype of control and Wnt5a CKO mice. Figure S4. Baseline osteoclast phenotype of control and Wnt5a cKO mice. Figure S5. Additional histopathological scoring of arthritis in Wnt5a CKO mice. Figure S6. Gene expression of cytokines and osteoclast genes in paws of control and Wnt5a CKO mice undergoing STIA. Figure S7. Characteristics of fusing osteoclasts in the absence and presence of Wnt5a-treated medium. Figure S8. Gene expression of osteoclast markers during BMDM differentiation to osteoclasts. Figure S9. Gene expression of NFATa1 markers during BMDM differentiation to osteoclasts. (PDF 4647 kb)

\footnotetext{
Abbreviations

ANOVA: Analysis of variance; BMDM: Bone marrow-derived macrophages; CAL: Calcitonin receptor; CAR2: Carbonic anhydrase 2; CIA: Collagen-induced arthritis; CLCN7: Chloride voltage-gated channel 7; CTSK: Cathepsin K: DAPI: 4',6-Diamidino-2-phenylindole; DCSTAMP: Dendrocyte-expressed seven transmembrane protein; DMARD: Disease-modifying anti-rheumatic drug; DMEM: Dulbecco's modified Eagle's medium; FBS: Fetal bovine serum; H\&E: Hematoxylin and eosin; HPF: High-powered field; HPRT: Hypoxanthine guanine phosphoribosyl transferase; IACUC: Institutional Animal Care and Use Committee; KO: Knockout; MCP1: Monocyte chemoattractant protein 1; MCSF: Macrophage colony-stimulating factor; MMP2: Matrix metalloproteinase 2; MMP9: Matrix metalloproteinase 9; MMP14: Matrix
} 
metalloproteinase 14; NFAT1c: Nuclear factor activating T cells $1 \mathrm{c}$ OPG: Osteoprotegerin; OSCAR: Osteoclast-associated immunoglobulin-like receptor; PMN: Polymononuclear; RA: Rheumatoid arthritis; RANKL: Signal receptor activator of nuclear factor-kB ligand; RCAN2: Regulator of calcineurin 2; ROR2: Receptor-tyrosine orphan receptor 2; STIA: Serum transfer-induced arthritis; TNFa: Tumor necrosis factor alpha; TRAP: Tartrate resistant acid phosphatase; Wnt5a: Wingless-type MMTV integration site family, member 5a

\section{Acknowledgements}

Not applicable.

\section{Funding}

This work was funded by National Institutes of Health (NIH) grants HL120160, HL131006, HL132564, and AG052160 to KW. SM was funded by a T32 grant HL007224. This work was supported by grants from the NIH to CMC (K01AR064313) and HP (AR064546, AR050250, AR054796, Al092490, HL108795), and funds provided to HP by the Mabel Green Myers Chair of Medicine. None of the funding bodies participated in the design of the study or in the collection, analysis, or interpretation of data or in writing the manuscript.

\section{Availability of data and materials}

The datasets supporting the conclusions of this article are included within the article (and its additional file(s)).

\section{Authors' contributions}

SM designed, performed, and analyzed the in vitro and in vivo experiments, and wrote and critically edited the manuscript. MAZ performed and analyzed the in vivo analysis. JJF performed and analyzed the in vivo analysis. JPD performed and analyzed the in vivo analysis. TA performed and analyzed the in vivo analysis. CC performed pathology assessment and analysis of the RA samples. JJ performed pathology assessment and analysis of the RA samples. GKH performed pathology assessment and analysis of the RA samples. FB performed and quantified in vitro analyses and staining. HP provided scientific support and critical editing of the manuscript. KW provided scientific support and critical editing of the manuscript. All authors read and approved the final manuscript and agree to be accountable for all aspects of the work

\section{Ethics approval and consent to participate}

Human subjects: not applicable, as there were no human subjects. Animals: all animal protocols were approved by the Boston University IACUC.

\section{Consent for publication}

Not applicable.

\section{Competing interests}

The authors declare that they have no competing interests.

\section{Publisher's Note}

Springer Nature remains neutral with regard to jurisdictional claims in published maps and institutional affiliations.

\section{Author details \\ ${ }^{1}$ Molecular Cardiology, Whitaker Cardiovascular Institute, Boston University School of Medicine, 700 Albany Street, W-611, Boston, MA 02118, USA. \\ ${ }^{2}$ Division of Rheumatology, Department of Medicine, Northwestern University, Feinberg School of Medicine, 240 E. Huron Street, McGaw M338 Chicago, IL, USA. ${ }^{3}$ University of Rochester Medical Center, School of Medicine and Dentistry, 601 Elmwood Ave, Box 665, Rochester, NY, USA. ${ }^{4}$ Renal Section, Boston University School of Medicine, 650 Albany St, Boston, MA, USA. ${ }^{5}$ Department of Pathology, Icahn School of Medicine at Mount Sinai, New York, NY 10029, USA.}

Received: 7 November 2016 Accepted: 27 June 2017 Published online: 19 July 2017

\section{References}

1. Koenders MI, van den Berg WB. Novel therapeutic targets in rheumatoid arthritis. Trends Pharmacol Sci. 2015;36(4):189-95.
2. McInnes IB, O'Dell JR. State-of-the-art: rheumatoid arthritis. Ann Rheum Dis. 2010;69(11):1898-906.

3. del Rincón ID, Williams K, Stern MP, Freeman GL, Escalante A. High incidence of cardiovascular events in a rheumatoid arthritis cohort not explained by traditional cardiac risk factors. Arthritis Rheum. 2001;44(12): 2737-45.

4. Meune C, Touzé E, Trinquart L, Allanore Y. Trends in cardiovascular mortality in patients with rheumatoid arthritis over 50 years: a systematic review and meta-analysis of cohort studies. Rheumatology (Oxford). 2009;48(10):1309-13.

5. Tanaka Y. Current concepts in the management of rheumatoid arthritis. Korean J Intern Med. 2016;31(2):210-8.

6. Niehrs C. The complex world of WNT receptor signalling. Nat Rev Mol Cell Biol. 2012;13(12):767-79.

7. Bretón-Romero R, Feng B, Holbrook M, Farb MG, Fetterman JL, Linder EA, Berk BD, Masaki N, Weisbrod RM, Inagaki E, et al. Endothelial dysfunction in human diabetes is mediated by Wnt5a-JNK signaling. Arterioscler Thromb Vasc Biol. 2016;36(3):561-9.

8. Farb MG, Karki S, Park SY, Saggese SM, Carmine B, Hess DT, Apovian C, Fetterman JL, Bretón-Romero R, Hamburg NM, et al. WNT5A-JNK regulation of vascular insulin resistance in human obesity. Vasc Med. 2016;21(6):489-96

9. Kikuchi R, Nakamura K, MacLauchlan S, Ngo DT, Shimizu I, Fuster JJ, Katanasaka Y, Yoshida S, Qiu Y, Yamaguchi TP, et al. An antiangiogenic isoform of VEGF-A contributes to impaired vascularization in peripheral artery disease. Nat Med. 2014;20(12):1464-71.

10. Fuster JJ, Zuriaga MA, Ngo DT, Farb MG, Aprahamian T, Yamaguchi TP, Gokce N, Walsh K. Noncanonical Wnt signaling promotes obesity-induced adipose tissue inflammation and metabolic dysfunction independent of adipose tissue expansion. Diabetes. 2015;64(4):1235-48.

11. Nakamura K, Sano S, Fuster JJ, Kikuchi R, Shimizu I, Ohshima K, Katanasaka Y, Ouchi N, Walsh K. Secreted frizzled-related protein 5 diminishes cardiac inflammation and protects the heart from ischemia/reperfusion injury. J Biol Chem. 2016;291(6):2566-75.

12. Ouchi N, Higuchi A, Ohashi K, Oshima Y, Gokce N, Shibata R, Akasaki Y, Shimono A, Walsh K. Sfrp5 is an anti-inflammatory adipokine that modulates metabolic dysfunction in obesity. Science. 2010;329(5990):454-7.

13. Halleskog C, Dijksterhuis JP, Kilander MB, Becerril-Ortega J, Villaescusa JC, Lindgren E, Arenas E, Schulte G. Heterotrimeric G protein-dependent WNT$5 \mathrm{~A}$ signaling to ERK1/2 mediates distinct aspects of microglia proinflammatory transformation. J Neuroinflammation. 2012;9:111.

14. Blumenthal A, Ehlers S, Lauber J, Buer J, Lange C, Goldmann T, Heine H, Brandt E, Reiling N. The Wingless homolog WNT5A and its receptor Frizzled5 regulate inflammatory responses of human mononuclear cells induced by microbial stimulation. Blood. 2006;108(3):965-73.

15. Pereira C, Schaer DJ, Bachli EB, Kurrer MO, Schoedon G. Wnt5A/CaMKII signaling contributes to the inflammatory response of macrophages and is a target for the antiinflammatory action of activated protein $\mathrm{C}$ and interleukin-10. Arterioscler Thromb Vasc Biol. 2008;28(3):504-10.

16. Rauner M, Stein N, Winzer M, Goettsch C, Zwerina J, Schett G, Distler JH, Albers J, Schulze J, Schinke T, et al. WNT5A is induced by inflammatory mediators in bone marrow stromal cells and regulates cytokine and chemokine production. J Bone Miner Res. 2012;27(3):575-85.

17. $\mathrm{Yu} \mathrm{CH}$, Nguyen $\mathrm{TT}$, Irvine $\mathrm{KM}$, Sweet MJ, Frazer $\mathrm{H}$, Blumenthal A. Recombinant Wnt3a and Wnt5a elicit macrophage cytokine production and tolerization to microbial stimulation via Toll-like receptor 4. Eur J Immunol. 2014;44(5):1480-90.

18. Sen M, Lauterbach K, El-Gabalawy H, Firestein GS, Corr M, Carson DA. Expression and function of wingless and frizzled homologs in rheumatoid arthritis. Proc Natl Acad Sci USA. 2000;97(6):2791-6.

19. Sen M, Chamorro M, Reifert J, Corr M, Carson DA. Blockade of Wnt-5A/ frizzled 5 signaling inhibits rheumatoid synoviocyte activation. Arthritis Rheum. 2001;44(4):772-81.

20. Maeda K, Kobayashi Y, Udagawa N, Uehara S, Ishihara A, Mizoguchi T, Kikuchi Y, Takada I, Kato S, Kani S, et al. Wnt5a-Ror2 signaling between osteoblast-lineage cells and osteoclast precursors enhances osteoclastogenesis. Nat Med. 2012;18(3):405-12.

21. Yamaguchi TP, Bradley A, McMahon AP, Jones S. A Wnt5a pathway underlies outgrowth of multiple structures in the vertebrate embryo. Development. 1999;126(6):1211-23.

22. Monach PA, Mathis D, Benoist C. The K/BxN arthritis model. Curr Protoc Immunol. 2008;Chapter 15:Unit 15.22. 
23. Scatizzi JC, Hutcheson J, Bickel E, Woods JM, Klosowska K, Moore TL, Haines GK, Perlman H. p21Cip1 is required for the development of monocytes and their response to serum transfer-induced arthritis. Am J Pathol. 2006;168(5): $1531-41$.

24. Scatizzi JC, Mavers M, Hutcheson J, Young B, Shi B, Pope RM, Ruderman EM, Samways DS, Corbett JA, Egan TM, et al. The CDK domain of p21 is a suppressor of IL-1 beta-mediated inflammation in activated macrophages. Eur J Immunol. 2009;39(3):820-5.

25. Misharin AV, Cuda CM, Saber R, Turner JD, Gierut AK, Haines GK, Berdnikovs S, Filer A, Clark AR, Buckley CD, et al. Nonclassical Ly6C(-) monocytes drive the development of inflammatory arthritis in mice. Cell Rep. 2014;9(2):591-604

26. Scatizzi JC, Bickel E, Hutcheson J, Haines GK, Perlman H. Bim deficiency leads to exacerbation and prolongation of joint inflammation in experimental arthritis. Arthritis Rheum. 2006;54(10):3182-93.

27. Scatizzi JC, Hutcheson J, Bickel E, Haines GK, Perlman H. Pro-apoptotic Bid is required for the resolution of the effector phase of inflammatory arthritis. Arthritis Res Ther. 2007;9(3):R49.

28. Perlman H, Georganas C, Pagliari LJ, Koch AE, Haines K, Pope RM. BCl-2 expression in synovial fibroblasts is essential for maintaining mitochondrial homeostasis and cell viability. J Immunol. 2000;164(10):5227-35.

29. Chen W, ten Berge D, Brown J, Ahn S, Hu LA, Miller WE, Caron MG, Barak LS, Nusse R, Lefkowitz RJ. Dishevelled 2 recruits beta-arrestin 2 to mediate Wnt5A-stimulated endocytosis of Frizzled 4. Science. 2003;301(5638):1391-4.

30. Yagi M, Miyamoto T, Sawatani Y, Iwamoto K, Hosogane N, Fujita N, Morita K, Ninomiya K, Suzuki T, Miyamoto K, et al. DC-STAMP is essential for cell-cell fusion in osteoclasts and foreign body giant cells. J Exp Med. 2005;202(3):345-51.

31. Wang B, Zinselmeyer BH, Runnels HA, LaBranche TP, Morton PA, Kreisel D, Mack M, Nickerson-Nutter C, Allen PM, Miller MJ. In vivo imaging implicates CCR2(+) monocytes as regulators of neutrophil recruitment during arthritis. Cell Immunol. 2012;278(1-2):103-12.

32. Wipke BT, Allen PM. Essential role of neutrophils in the initiation and progression of a murine model of rheumatoid arthritis. J Immunol. 2001; 167(3):1601-8.

33. Duffau P, Menn-Josephy H, Cuda CM, Dominguez S, Aprahamian TR, Watkins AA, Yasuda K, Monach P, Lafyatis R, Rice LM, et al. Promotion of inflammatory arthritis by interferon regulatory factor 5 in a mouse model. Arthritis Rheumatol. 2015;67(12):3146-57.

34. Jung YS, Lee HY, Kim SD, Park JS, Kim JK, Suh PG, Bae YS. Wnt5a stimulates chemotactic migration and chemokine production in human neutrophils. Exp Mol Med. 2013;45, e27.

35. Logan CY, Nusse R. The Wnt signaling pathway in development and disease. Annu Rev Cell Dev Biol. 2004;20:781-810.

36. Kurayoshi M, Yamamoto H, Izumi S, Kikuchi A. Post-translational palmitoylation and glycosylation of Wnt-5a are necessary for its signalling. Biochem J. 2007:402(3):515-23.

37. Bellon M, Ko NL, Lee MJ, Yao Y, Waldmann TA, Trepel JB, Nicot C. Adult Tcell leukemia cells overexpress Wnt5a and promote osteoclast differentiation. Blood. 2013;121(25):5045-54.

38. Bilkovski R, Schulte DM, Oberhauser F, Gomolka M, Udelhoven M, Hettich MM, Roth B, Heidenreich A, Gutschow C, Krone W, et al. Role of WNT-5a in the determination of human mesenchymal stem cells into preadipocytes. J Biol Chem. 2010;285(9):6170-8.

39. Wang Y, Li YP, Paulson C, Shao JZ, Zhang X, Wu M, Chen W. Wnt and the Wnt signaling pathway in bone development and disease. Front Biosci (Landmark Ed). 2014:19:379-407.

40. Helming L, Gordon S. Molecular mediators of macrophage fusion. Trends Cell Biol. 2009;19(10):514-22.

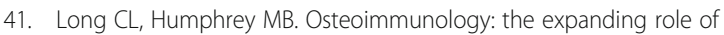
immunoreceptors in osteoclasts and bone remodeling. Bonekey Rep. 2012; 1:59-65.

42. Rajasekaran N, Illges H. Matrix Metalloproteinase MMP-9 Promotes K/BxN Serum Induced Arthritis in Mice. Open J Rheumatol Autoimmune Dis. 2014; 4(1):22-8. doi:10.4236/ojra.2014.41003.

43. MacLauchlan S, Skokos EA, Meznarich N, Zhu DH, Raoof S, Shipley JM, Senior RM, Bornstein P, Kyriakides TR. Macrophage fusion, giant cell formation, and the foreign body response require matrix metalloproteinase 9. J Leukoc Biol. 2009;85(4):617-26.

44. Chabot V, Reverdiau P, lochmann S, Rico A, Sénécal D, Goupille C, Sizaret PY, Sensebé L. CCL5-enhanced human immature dendritic cell migration through the basement membrane in vitro depends on matrix metalloproteinase-9. J Leukoc Biol. 2006;79(4):767-78.
45. Wucherpfennig AL, Li YP, Stetler-Stevenson WG, Rosenberg AE, Stashenko P. Expression of $92 \mathrm{kD}$ type IV collagenase/gelatinase B in human osteoclasts. J Bone Miner Res. 1994;9(4):549-56.

46. Kusano K, Miyaura C, Inada M, Tamura T, Ito A, Nagase H, Kamoi K, Suda T. Regulation of matrix metalloproteinases (MMP-2, -3, -9, and -13) by interleukin-1 and interleukin-6 in mouse calvaria: association of MMP induction with bone resorption. Endocrinology. 1998;139(3):1338-45.

47. Asquith DL, Miller AM, Hueber AJ, Liew FY, Sattar N, Mclnnes IB. Apolipoprotein E-deficient mice are resistant to the development of collagen-induced arthritis. Arthritis Rheum. 2010;62(2):472-7.

48. Postigo J, Genre F, Iglesias M, Fernández-Rey M, Buelta L, Carlos RodríguezRey J, Merino J, Merino R. Exacerbation of type II collagen-induced arthritis in apolipoprotein E-deficient mice in association with the expansion of Th1 and Th17 cells. Arthritis Rheum. 2011;63(4):971-80.

49. Archer AM, Saber R, Rose S, Shaffer A, Misharin AV, Tsai F, Haines lii GK, Dominguez S, Eren M, Vaughan DE, et al. ApoE deficiency exacerbates the development and sustainment of a semi-chronic K/BxN serum transferinduced arthritis model. J Transl Med. 2016;14(1):170.

50. Rose S, Eren M, Murphy S, Zhang H, Thaxton CS, Chowaniec J, Waters EA, Meade TJ, Vaughan DE, Perlman H. A novel mouse model that develops spontaneous arthritis and is predisposed towards atherosclerosis. Ann Rheum Dis. 2013;72(1):89-95.

51. Catalán V, Gómez-Ambrosi J, Rodríguez A, Pérez-Hernández Al, Gurbindo J, Ramírez B, Méndez-Giménez L, Rotellar F, Valentí V, Moncada R, et al. Activation of noncanonical Wnt signaling through WNT5A in visceral adipose tissue of obese subjects is related to inflammation. J Clin Endocrinol Metab. 2014;99(8):E1407-17.

52. Versini M, Jeandel PY, Rosenthal E, Shoenfeld Y. Obesity in autoimmune diseases: not a passive bystander. Autoimmun Rev. 2014;13(9):981-1000.

53. Feng J, Chen Q, Yu F, Wang Z, Chen S, Jin Z, Cai Q, Liu Y, He J. Body mass index and risk of rheumatoid arthritis: a meta-analysis of observational studies. Medicine (Baltimore). 2016;95(8), e2859.

54. Del Prete A, Salvi V, Sozzani S. Adipokines as potential biomarkers in rheumatoid arthritis. Mediators Inflamm. 2014;2014:425068.

55. Otero M, Lago R, Gomez R, Lago F, Dieguez C, Gómez-Reino JJ, Gualillo O. Changes in plasma levels of fat-derived hormones adiponectin, leptin resistin and visfatin in patients with rheumatoid arthritis. Ann Rheum Dis. 2006;65(9):1198-201.

56. Yoshino T, Kusunoki N, Tanaka N, Kaneko K, Kusunoki Y, Endo H, Hasunuma $\mathrm{T}$, Kawai S. Elevated serum levels of resistin, leptin, and adiponectin are associated with C-reactive protein and also other clinical conditions in rheumatoid arthritis. Intern Med. 2011;50(4):269-75.

57. Olama SM, Senna MK, Elarman M. Synovial/serum leptin ratio in rheumatoid arthritis: the association with activity and erosion. Rheumatol Int. 2012;32(3):683-90.

58. Giles JT, van der Heijde DM, Bathon JM. Association of circulating adiponectin levels with progression of radiographic joint destruction in rheumatoid arthritis. Ann Rheum Dis. 2011;70(9):1562-8.

59. Bokarewa M, Nagaev I, Dahlberg L, Smith U, Tarkowski A. Resistin, an adipokine with potent proinflammatory properties. J Immunol. 2005;174(9): 5789-95.

\section{Submit your next manuscript to BioMed Central and we will help you at every step:}

- We accept pre-submission inquiries

- Our selector tool helps you to find the most relevant journal

- We provide round the clock customer support

- Convenient online submission

- Thorough peer review

- Inclusion in PubMed and all major indexing services

- Maximum visibility for your research

Submit your manuscript at www.biomedcentral.com/submit 